\title{
NUEVOS REGISTROS DE SPHYRAENA (PERCIFORMES: SPHYRAENIDAE) PARA EL NEÓGENO DE CUBA Y LA ESPAÑOLA
}

\author{
Lázaro William Viñola López ${ }^{1}$, Reinaldo Rojas Consuegra ${ }^{2}$ y Osvaldo Jiménez Vázquez ${ }^{3}$ \\ ${ }^{1}$ Earth Sciences Department, Montana State University, Bozeman, \\ MT, 59717, USA lazarowillian94@gmail.com \\ ${ }^{2}$ Instituto de Investigaciones del Petróleo. La Habana, Cuba. \\ ${ }^{3}$ Laboratorio de Arqueozoología, Gabinete de Arqueología, \\ Oficina del Historiador de la Ciudad de La Habana, Cuba.
}

\section{RESUMEN}

Tras el estudio de una amplia muestra de peces fósiles del terciario de Cuba y la Española, hemos identificado restos que corresponden al género Sphyraena, previamente reportado para el plioceno de Cuba. En el presente artículo extendemos la distribución temporal y espacial de barracuda en el Caribe con tres nuevas localidades del neógeno de Cuba y una para la Española.

Palabras clave: Sphyraena sp., dientes fósiles, Cuba, la Española, mioceno.

\section{NEW RECORDS OF SPHYRAENA (PERCIFORMES: SPHYRAENIDAE) FOR THE NEOGENE OF CUBA AND HISPANIOLA}

\section{ABSTRACT}

The study of an array of fossils of fishes from the tertiary of Cuba and the Hispaniola has allow us to identify several teeth of Sphyraena. This genus was previously recorded for the pliocene of western Cuba. Here we extend the temporal and spatial distribution of barracuda in the Caribbean, with three new localities in Cuba and one in the Hispaniola.

Keywords: Sphyraena sp., fossil teeth, Cuba, Hispaniola, Miocene.

\section{INTRODUCCIÓN}

En el registro fósil del neógeno antillano los peces están relativamente bien representados, sin embargo son pocas las publicaciones sobre el tema y la mayoría de ellos permanecen sin estudiar (Iturralde-Vinent et al., 1996, 1998; Diaz y Rojas-Consuegra, 2009; Viñola y Rojas-Consuegra, 2016). En sentido general, la mayor parte de colectas y estudios han estado dirigidos hacia los condríctios, de los cuales se conoce el mayor número de especies, mientras los osteíctios han recibido poca atención. Hasta el momento, solo tres géneros y cinco especies fósiles de osteíctios han sido identificados para Cuba: dos especies de pez guanábana (Diodon circunflexus y D. scillae), una barracuda o picúa (Sphyraena cf. barracuda) y dos especies del género Sparus (Iturralde-Vinent y Case, 1998; Vázquez y Grau, 2004; Díaz y Rojas-Consuegra, 2009; Viñola y Rojas-Consuegra, 2016).

El género Sphyraena tiene una distribución amplia en mares tropicales (Sylva, 1990) y de este se conocen abundantes restos fósiles, hasta de edad eoceno Inferior (Kumar et al., 2007 y Mehling, 2007), aunque la mayor parte del registro fósil se concentra en rocas del neógeno (Cervigón, 1993; Gasparini y Floeter, 2001). En el Caribe, el único registro fósil de Sphyraena cf. barracuda se conoce de un depósito del plioceno en el Abra del Yumurí en Matanzas, Cuba. En ese artículo, Díaz y Rojas-Consuegra (2009) reportan nueve dientes de barracuda y realizan inferencias tafonómicas, aportando 
elementos sobre las condiciones paleoambientales del depósito. El reporte de Sphyraena en el Abra de Figueroa, por Rojas-Consuegra y Viñola (2013), es un error toponímico, puesto que en realidad se referían a la Abra del Yumurí.

\section{OBJETIVO}

-Extender el registro temporal y espacial de Sphyraena en las Antillas, dando a conocer cuatro nuevas localidades del mioceno, tres en Cuba y una en la Española.

\section{MATERIALES Y MÉTODOS}

La identificación de los ejemplares se realizó mediante la comparación directa con especímenes actuales de Sphyraena barracuda, colectados en diferentes puntos de la plataforma insular cubana. Dichas piezas se encuentras depositadas en el Museo Nacional de Historia Natural de Cuba (un ejemplar) y en la colección personal Lázaro Viñola (CLV; tres ejemplares del sur de la Cienaga de Zapata). Así mismo, las descripciones e ilustraciones publicadas por Díaz y Rojas-Consuegra (2009) fueron empleados para identificar los especímenes.

Material examinado. Se utilizó una serie de quince dientes depositados en la colección paleontológica del Museo Nacional de Historia Natural de Cuba (MNHNCu) y en la colección personal Lázaro Viñola (CLV). Los ejemplares MNHNCu-71.1, 71.2, proceden de afloramientos al norte de Thomonde, Haití, colectados por Manuel A. Iturralde-Vinent, Reinaldo Rojas y Stephen Díaz, en el año 2001. Cuatro ejemplares, MNHNCu-71.3, 71.4, CLV-2454, CLV-2537, fueron colectados por Ricardo Figueroa Mendosa entre 2013 y 2014 en la Cantera J-4, Jagüey Grande, Matanzas, Cuba. Ocho ejemplares: MNHNCu-71.6, 71.7, 71.8, 71.9, 71.10, 71.11, 71.12, 71.13, proceden de cantera La Pendencia, Alturas de Bejucal, en la provincia de Artemisa, Cuba; obtenidos por Osvaldo Jiménez Vázquez, Oscar Sánchez y Elier Fonseca, entre 1997 y 1999. El ejemplar MNHNCu (71.14) fue colectado por Oscar Sánchez, en 1996, en una cantera en la ladera sur del Cacahual, a 400 metros de Bejucal por la carretera del Rincón, en la mencionada provincia.

\section{CONTEXTO GEOLÓGICO}

Thomonde. Los especímenes MNHNCu-71.1, 71.2 fueron colectados en un corte en la carretera al norte de Thomonde, Haití. El depósito está constituido por arcillas grises calcáreas con restos de corales, moluscos, dientes de peces óseos, dientes de tiburones y mantas, fragmento de tortuga y ámbar (Iturralde-Vinent, 2001). Según este autor las rocas son del mioceno, semejantes a las de la Formación Lagunitas de Cuba y la Formación Cibao de Puerto Rico. Los sedimentos de este corte pertenecen a la Formación Maissade.

Canteras J-4. Esta localidad está formada por rocas de la Formación Colón, de edad oligoceno tardío-mioceno temprano. Todos los especímenes provienen de la sección superior del depósito, considerándose miocénicos. En general, corresponde a calizas biodetríticas, de color blanco o crema de grano muy fino y con abundantes restos de crustáceos, dientes de tiburones, restos de peces óseos, tortugas, cocodrilos y sirénidos (Varela y Rojas-Consuegra, 2009; Varela y Schweitzer, 2011; Varela y Rojas-Consuegra, 2011, 2011a, 2011b; Viñola y Rojas Cosuegra, 2016). Varela y Rojas-Cosuegra(2011), Viñola y Rojas Cosuegra(2016)y Varela y Rojas-Cosuegra (2011), basándose en la constitución geológica de esta localidad y en 
la asociación de crustáceos fósiles, propusieron que el ambiente deposicional debió haber sido intermareal-deltaico, de poca profundidad.

Cantera La Pendencia y Cantera en Bejucal. Ambas localidades se abren en rocas de la Formación Husillo del mioceno Inferior. Esta formación tiene una amplia distribución en Artemisa, La Habana y Mayabeque. La misma se caracteriza litológicamente por poseer calizas biógenas, biodetríticas, de grano fino, calizas biohérmicas y calizas fragmentarias, también calizas arcillosas. En general son masivas, pero hay secciones, desde bien hasta regularmente estratificadas. Representa una facies de mar somero, isócrona con facies de mar poco profundo de la Formación Jaruco, depositadas durante un proceso transgresivo; donde se formaron biohermas y arrecifes locales (Iturralde-Vinent, 2012). La asociación fosilífera se encuentra compuesta por equinodermos, poríferos, moluscos bivalvos y gasterópodos, tubos de poliquetos, dientes de tiburones, peces óseos y fragmentos de un quelonio indeterminado (Aranda-Manteca et al., 2011).

\section{TAXONOMÍA}

\section{Orden Perciformes}

Familia Sphyraenidae Walbaum, 1792

Género Sphyraena Artedis, 1793

Descripción. Tras el estudio de los especímenes hemos asignado los mismos al género Sphyraena, basados en un conjunto de elementos diagnósticos (ver Gillete, 1984; Díaz y Rojas-Consuegra, 2009) como: forma triangular de los dientes con ausencia de raíz, comprimidos medio lateralmente, llegando a ser muy rectos; poseen bordes completamente aserrados casi imperceptibles a simple vista, pero al microscopio se observan como pequeñas concavidades que coinciden en ambas caras. Además poseen una línea ligeramente deprimida cercana a los bordes aserrados (dentículos), que los recorre paralelamente en su totalidad, así como numerosos "alineamientos cuasiparalelos" (ver Franco y Rojas-Consuegra, 2009), en la corona desde la región basal hasta la apical, más marcados en la primera, disminuyendo de intensidad paulatinamente. Este carácter es más notable en los dientes delanteros de la serie cráneo-mandibular; en sentido general presentan una morfología uniforme. En la Tabla I se ofrecen las medidas de parte de la muestra.

TABLA I.

Medidas de los ejemplares de Sphyraena colectados en rocas del mioceno de Cuba y la Española (cm).

\begin{tabular}{|l|ccc|}
\hline No. & Longitud total & Anchura basal & Profundidad basal \\
\hline Serie anterior & - & - & - \\
\hline MNHNCu-71.3 & - & 5.31 & 2.72 \\
\hline Serie medio-terminal & - & - & - \\
\hline MNHNCu-71.1 & - & 8.11 & 3.08 \\
\hline MNHNCu-71.2 & - & 5.31 & 2.17 \\
\hline MNHNCu-71.6 & 8.09 & 4.97 & 2.77 \\
\hline MNHNCu - & 7.85 & - & 2.27 \\
\hline MNHNCu - & 6.28 & - & 1.52 \\
\hline MNHNCu - & 5.11 & - & 1.20 \\
\hline CLV-2454 & 8.42 & 5.61 & 1.92 \\
\hline
\end{tabular}


Once de los ejemplares (MNHNCu-71.1, 71.2, 71.4, 71.6, 71.9, 71.10, 71.11, 71.12, 71.13, 71.14, CLV-2454,) corresponden morfológicamente a la serie medio-terminal de los dientes cráneo-mandibulares (Díaz y Rojas-Consuegra, 2009), caracterizados por ser simétricos al trazarse un eje longitudinal tanto en los planos linguales como labiales, con ambos bordes aserrados en su totalidad, la cara lingual convexa y la labial algo más aplanada.

Cuatro de los ejemplares (MNHNCu-71.3, 71.7, 71.8 y CLV-2537), pertenecen a la región anterior de la serie cráneo-mandibular, asimétricos bilateralmente, presentando dentículos en el borde anterior y una línea deprimida paralela a estos como se mencionó anteriormente. El borde posterior se caracteriza por ser redondeado y poseer, cercano a la cúspide, una estructura sobresaliente en forma de ala con dentículo y la línea paralela. Lamentablemente, la mayor parte de los especímenes poseen las cúspides fracturadas; aunque en el ejemplar CLV-Sn se puede observar la sección más proximal del mismo.

\section{DISCUSIÓN}

Con estos nuevos registros se amplían nuestros conocimientos sobre la distribución de Sphyraena en el Caribe durante el mioceno. A pesar de no encontrar diferencias morfológicas con los ejemplares actuales de Sphyraena barracuda, decidimos identificar los especímenes a nivel de género, puesto que no contamos con muestras comparativas de otras de las especies extintas del neógeno.

Díaz y Rojas-Consuegra (2009), plantearon la posibilidad que los ejemplares estudiados por ellos pudieran provenir de rocas más antiguas, reelaboradas y redepositadas en la formación El Abra. Este criterio se basa, en la presencia de entidades de cuerpos geológicos del cretácico y del neógeno encontrados allí (Díaz y Rojas-Consuegra, 2009); así como la presencia de microestrías superficiales provocadas por transporte postdeposicional en el cauce del río (Díaz y Rojas-Consuegra, 2009). En la muestra estudiada no se observó este tipo alteración tafonómica, en parte dado porque fueron colectadas en ambientes que debieron ser menos energéticos y con sedimentos de menor granulometría y dureza. Sin embargo, tres ejemplares (MNHNCu-71.11, 71.12; CLV-2454) exhiben microfracturas en la corona y en los bordes acerrados. Estas mismas alteraciones fueron observadas en ejemplares modernos del género, por lo cual inferimos que sean resultado de la actividad trófica, aunque no se puede descartar la posibilidad de que hayan sido producidas por transporte. Otra de las alteraciones tafonómica es la formación de bandas de colores (MNHNCu-71.1, 71.2, 71.9), de la región basal a la apical (fig. 1C).

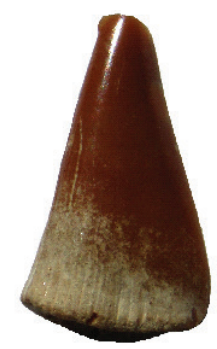

A

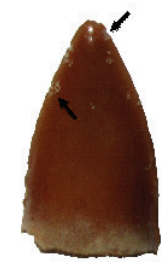

$\mathrm{B}$

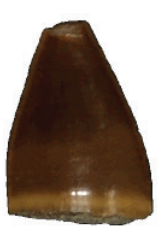

$\mathrm{C}$

FIGURA 1. A-C. Dientes de la serie cráneo-mandibular de Sphyraena sp. A, región anterior (CLV-2537). B y C, región medio-posterior (CLV-2455 y $\mathrm{MNHNCu}-71.1$ respectivamente). Las flechas en $\mathrm{B}$ indican la presencia de microfracturas en la cúspide y en bordes acerrados. Obsérvese en $\mathrm{C}$ bandas de diferente coloración debidas a diferentes grados de recristalización y/o sustitución. 
Este bandeamiento lo interpretamos como posible recristalización y/o sustitución (Fernández-López, 2000), comenzando por la región basal donde está expuesta la dentina e interactúa en mayor grado con el medio. Estos nuevos registros demuestran que Sphyraena ha estado presente en parte del territorio que hoy ocupa el Caribe insular desde el Mioceno Inferior, habitando en ambientes similares a los que ocupan hoy las especies de este género.

\section{AGRADECIMIENTOS}

Agradecemos a Ricardo Figueroa, Elier Fonseca, Oscar Sánchez, Stephen Diaz y Manuel Irurralde por la colecta de los especímenes utilizados en e presente estudio. Así mismo, a Johanset Orihuela por la revisión crítica del artículo.

\section{LITERATURA CITADA}

Aranda-Manteca F. J., R. Rojas-Consuegra y O. Jiménez. 2011. Carcharhinidae de Cuba y Haití en la Colección del Museo Nacional de Historia Natural de Cuba. Resumen en XII Congreso Nacional de Paleontología (SOMEXPAL), 22 al 25 de Febrero de 2011, Benemérita Universidad de Puebla.

Cervigón, F. 1993. Los peces marinos de Venezuela. Vol. 2. Fundación Científica Los Roques, Caracas, Venezuela, p. 497.

Díaz Franco, S. y R. Rojas-Consuegra. 2009. Dientes fósiles de Sphyraena (Perciformes: Sphyraenidae) en el Terciario de Cuba occidental. Solenodon, 8: 124-129.

Fernández-López, S. R. 2000. Temas de Tafonomía. Departamento de Paleontología, Universidad Complutense de Madrid, 167 pp.

Gasparini, J. L. y S. R. Floeter. 2001. The shore fishes of Trinidad Island, western South Atlantic. J. Nat. Hist., 35: 1639-1656.

Gillete, D. D. 1984. A marine ichthyofauna from the Miocene of Panamá, and the Tertiary Caribbean faunal province. J. Vert. Paleontol., 4 (2): 172-186.

Iturralde-Vinent, M. 2001. Geology of the Amber-Bearing Deposits of the Greater Antilles. Caribbean Journal of Science, 37 (3/4): 141-167.

Iturralde-Vinent, M. A. 2012. (Editor). Compendio de Geología de Cuba y del Caribe. Segunda Edición. DVD-ROM. Editorial CITMATEL, La Habana, Cuba.

Iturralde-Vinent, M. A. y G. R. Case. 1998. First report of the fossil fish, Diodon (Family Diodontidae) from the Miocene of Cuba. Rev. Soc. Mexicana Paleontol., 8 (2):123-126.

Iturralde-Vinent, M., G. Hubbell y R. Rojas. 1996. Catalogue of cuban fossil Eslamobranchii (Paleocene- Pliocene) and Paleogeographic implications of their Lower to Middle Miocene occurrence. The journal of the Geological Society of Jamaica, 31: 7-21.

Iturralde-Vinent, M., C. L. Mora, R. Rojas y M. R. Gutiérrez. 1998. Myliobatidae(Elasmobranchii: Batomorphii) del Terciario de Cuba. Rev. Soc. Mex. Paleontología, 8 (2): 135-145. 
Kuiter, R. H. y T. T. onozuka. 2001. Pictorial guide toIndonesianreeffishes. Parte 3. JawfishesSunfishes, Opistognathidae-Molidae. Zoonetics, Australia, 623-893.

Kumar, K., R. S. Rana y H. Singh. 2007. Fishes of the Khuiala Formation (Early Eocene) of the Jaisalmer Basin, Western Rajasthan, India. Current Science, 93 (4): 553-559.

Mehling, C. 2007. Fossil. Publicher San Diego, California. 320 pp.

Rojas-Consuegra, R. y L. W. Viñola-López. 2013. La región paleontológica Matanzas: un caso patrón. Revista electrónica Savia, 3: 18. Enero 2013. ISSN 2224-9532.

Sylva, D . P .1990. Sphyraenidae. In Check-list of the fishes of the eastern tropical Atlantic, J. C. Quero, J. C. Hureau, C. Karrer, A. Post y L. Saldanha, eds., (Clofeta), JNICT, Lisbon, SEI, París, y Unesco, París, 2: 860-864.

Varela, C. y R. Rojas-Consuegra. 2009. Crustáceos (Decapoda: Brachyura) fósiles de Cuba. Solenodon, 8: 118-123.

Varela, C. y R. Rojas-Consuegra. 2011. El registro fósil de los crustáceos decápodos (Arthropoda, Crustacea) marinos de Cuba. En: Resumen y Trabajo de la III Convención sobre Ciencias de La Tierra (Geociencia' 2011). VI Congreso Cubano de Geología, Estratigrafía y Paleontología. Memorias en CD-ROM, GEO2-P7, 10 pp. La Habana. ISBN 978-959-7117-30-8.

Varela, C. y R. Rojas-Consuegra. 2011a. Nueva especie de Eriosachila Blow y Manning, 1996 (Crustacea: Decapoda), de la Formación Colón, Cuba. Novitates Caribbea, 4: 17-20.

Varela, C. and R. Rojas-Consuegra. 2011b. Crustáceos fósiles (Decapoda: Brachyura), de la Formación Colón, Matanzas, Cuba. Solenodon, 9: 66-70.

Varela, C and Carrie E. Schweitzer 2011. A new genus and new species of Portunidae Rafinesque, 1815 (Decapoda, Brachyura) from the Colón Formation, Cuba. Bulletin of the Mizunami Fossil Museum, no. 37: 13-16.

Vázquez de la Torre, I. y E. Grau González-Quevedo. 2004. Descubren pez fósil del mioceno en Matanzas. In Noti-Cem. Rev. Electrónica (1861: Revista de Espeleología y Arqueología), 1:37.

Viñola-López, L. W. y Rojas-Consuegra, R. 2016. Distribución del género Sparus (perciforme: sparidae) en el terciario de cuba occidental. Revista Geológica de América Central, (54): 57-66. 